\title{
Higher predation risk for insect prey at low latitudes and elevations
}

\author{
Tomas Roslin, ${ }^{1,2 *}$ Bess Hardwick, ${ }^{2}$ Vojtech Novotny, ${ }^{3,4,5}$ William K. Petry, ${ }^{6,7}$ \\ Nigel R. Andrew, ${ }^{8}$ Ashley Asmus, ${ }^{9}$ Isabel C. Barrio, ${ }^{10,11}$ Yves Basset, ${ }^{3,4,12}$ \\ Andrea Larissa Boesing, ${ }^{13}$ Timothy C. Bonebrake, ${ }^{14}$ Erin K. Cameron, ${ }^{15,16}$ Wesley Dáttilo, ${ }^{17}$ \\ David A. Donoso, ${ }^{18}$ Pavel Drozd, ${ }^{19}$ Claudia L. Gray, ${ }^{20,21}$ David S. Hik, ${ }^{10}$ Sarah J. Hill, ${ }^{8}$ \\ Tapani Hopkins, ${ }^{22}$ Shuyin Huang, ${ }^{23}$ Bonny Koane, ${ }^{5}$ Benita Laird-Hopkins, ${ }^{12}$ \\ Liisa Laukkanen, ${ }^{24}$ Owen T. Lewis, ${ }^{21}$ Sol Milne,${ }^{25}$ Isaiah Mwesige,${ }^{26}$ Akihiro Nakamura, ${ }^{23}$ \\ Colleen S. Nell, ${ }^{6}$ Elizabeth Nichols, ${ }^{13,27}$ Alena Prokurat, ${ }^{28}$ Katerina Sam, ${ }^{3,4}$ \\ Niels M. Schmidt, ${ }^{29,30}$ Alison Slade, ${ }^{31}$ Victor Slade, ${ }^{31}$ Alžběta Suchanková, ${ }^{19}$ Tiit Teder, ${ }^{32}$ \\ Saskya van Nouhuys, ${ }^{15}$ Vigdis Vandvik, ${ }^{33}$ Anita Weissflog, ${ }^{34}$ \\ Vital Zhukovich, ${ }^{28}$ Eleanor M. Slade ${ }^{2,21,35}$
}

Biotic interactions underlie ecosystem structure and function, but predicting interaction outcomes is difficult. We tested the hypothesis that biotic interaction strength increases toward the equator, using a global experiment with model caterpillars to measure predation risk. Across an 11,660-kilometer latitudinal gradient spanning six continents, we found increasing predation toward the equator, with a parallel pattern of increasing predation toward lower elevations. Patterns across both latitude and elevation were driven by arthropod predators, with no systematic trend in attack rates by birds or mammals. These matching gradients at global and regional scales suggest consistent drivers of biotic interaction strength, a finding that needs to be integrated into general theories of herbivory, community organization, and life-history evolution.

I $\mathrm{t}$ is widely accepted that species diversity increases toward the tropics (1). This gradient is so ubiquitous that it has been called one of the fundamental laws in ecology (2). However, whether this latitudinal variation in diversity is paralleled by similar gradients in the intensity of biotic interactions, both antagonistic and mutualistic (3-9), remains unclear.

A widespread view is that biotic interactions become increasingly strong at lower latitudes (10-12). However, accumulating evidence [e.g., $(7,8,13,14)]$ suggests that when critically examined, this pattern may be weak, absent, or even reversed. Part of this complexity arises because large-scale patterns are usually pieced together from data obtained by a variety of methods and protocols [e.g., $(7,15,16)]$. Here we use a simple, uniform protocol to quantify ecologically impor- tant patterns systematically at a global scale $(17,18)$. Specifically, we assess predation risk using the attack rate on model caterpillars (Fig. 1) for which the identity of the attacker may be determined (19). This method has been shown to provide accurate estimates of predator activity at individual sites and across local gradients $(20,21)$. By applying a consistent method at a global level, our study provides a rigorously controlled estimate of latitudinal patterns in predation strength.

Building on general theory $(3,10,11)$, we hypothesize that overall biotic interaction strength increases toward the equator. Many ecological factors that change with latitude also change with elevation, and thus it is important to control for elevational variation when quantifying latitudinal signals in predation rates. Moreover, by testing for congruence between latitudinal and elevational predation patterns, we can begin to identify candidate mechanisms underlying predation rates.

Regardless of where high predation rates are found, depredation of herbivores is predicted to have broad ecological and evolutionary consequences across trophic levels. Stronger predation on herbivores directly affects the abundance and traits of herbivores (22-24), but also indirectly affects the abundance and traits of plants through trophic cascades $(25,26)$. Gradients in interaction strength thus provide a foundation for understanding global patterns in ecosystem processes (e.g., herbivory and primary production), ecosystem services (e.g., carbon storage and crop yields), and how long-term environmental change may affect biodiversity.

Predation rates reflect the sum of attacks by several different predator groups, each of which may show a different latitudinal pattern. We expect predation by ectothermic predators such as arthropods to be strongly controlled by local abiotic conditions. Indeed, temperature has been identified as the dominant abiotic factor directly affecting ectothermic insects $(27,28)$, and in experiments, insect performance generally improves with temperature (29). An increase in the intensity of insect-mediated biotic interactions with increasing mean temperature is also supported by the higher folivory rates observed during warmer geological periods $(30,31)$. Thus, we expect increased attack rates by arthropod predators at low latitude and low elevation. By contrast, endotherms such as insectivorous birds may redistribute themselves through migration in response to local food availability (32). This is expected to homogenize predation rates across latitude and elevation or elevate predation rates in their higher-latitude breeding grounds.

To test these hypotheses, we systematically measured predation risk by monitoring the fates of 2879 model caterpillars over 4 to 18 days, resulting in a total of 12,694 caterpillar days (table S1). These caterpillars were molded from green plasticine, shaped in the posture of common generalist caterpillars, and deployed within five plots at each of 31 sites along a latitudinal gradient spanning from $30.4^{\circ} \mathrm{S}$ to $74.3^{\circ} \mathrm{N}$, and an elevation gradient spanning from 0 to 2100 meters

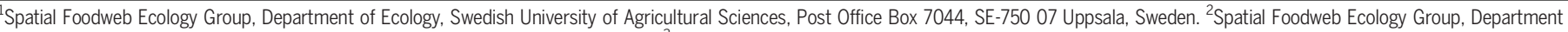

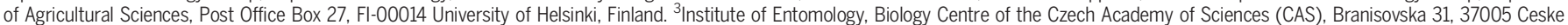
Budejovice, Czech Republic. ${ }^{4}$ Department of Zoology, Faculty of Science, University of South Bohemia, Branisovska 1760, 37005 Ceske Budejovice, Czech Republic. ${ }^{5}$ The New Guinea Binatang

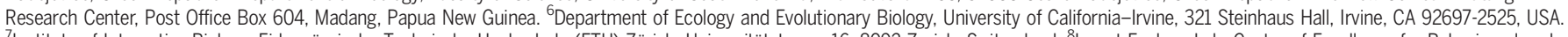

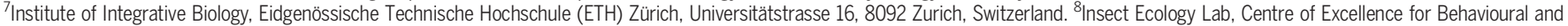

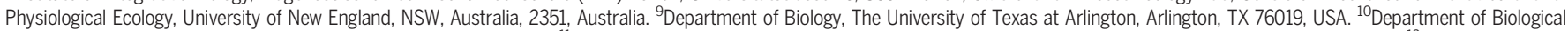

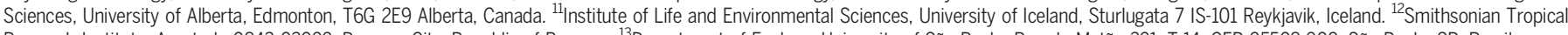
Research Institute, Apartado 0843-03092, Panama City, Republic of Panama. ${ }^{13}$ Department of Ecology, University of São Paulo, Rua do Matão 321, T-14, CEP 05508-900, São Paulo, SP, Brazil.

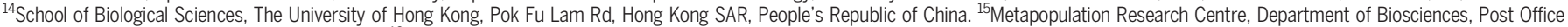
Box 65, Fl-00014, University of Helsinki, Finland. ${ }^{16}$ Center for Macroecology, Evolution and Climate Change, Natural History Museum of Denmark, University of Copenhagen, Universitetsparken 15,

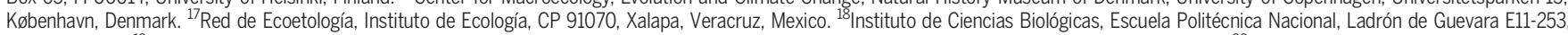
Quito, Ecuador. ${ }^{19}$ University of Ostrava, Faculty of Science-Department of Biology and Ecology, Chittussiho 10, 71000 Slezská Ostrava, Czech Republic. ${ }^{20}$ Evolutionarily Distinct and Globally

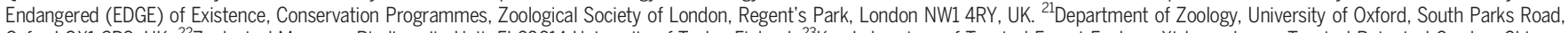

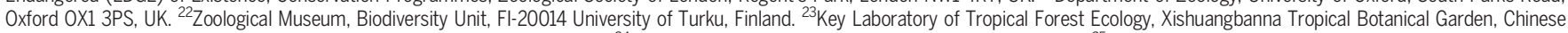

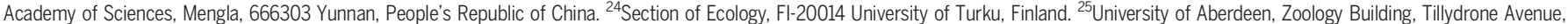

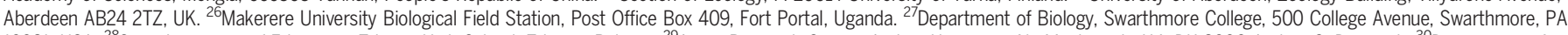

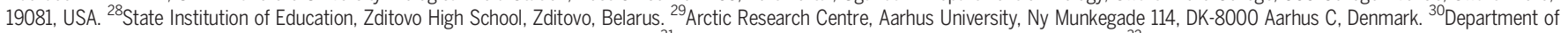

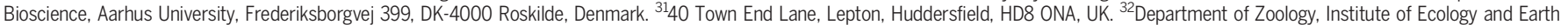
Sciences, University of Tartu, EE-51014 Tartu, Estonia. ${ }^{33}$ Department of Biology, University of Bergen, Post Office Box 7800, 5020 Bergen, Norway. ${ }^{34}$ Department of Plant Ecology, University of Bayreuth, 95440 Bayreuth, Germany. ${ }^{35}$ Lancaster Environment Centre, University of Lancaster, Lancaster, UK.

*Corresponding author. Email: tomas.roslin@slu.se
} 
above sea level (masl). Whenever an attack was scored, the caterpillar in question was removed without replacement. We used generalized linear mixed-effects models (33) to quantify the effects of latitude and elevation on biotic interaction strength, measured as the probability of a caterpillar being attacked per day exposed [for further validation of this response, see (33)].

Consistent with our predictions, we found that predation rates were highest at the equator and decreased significantly toward the poles (Fig. 1A; $F_{1,27.8}=10.28, P=0.003$ ). For every $1^{\circ}$ of latitude away from the equator, the daily odds of a caterpillar being attacked decreased by $2.7 \%$ (odds ratio
0.973 , confidence limits 0.959 to 0.987 ; Fig. 1A). Thus, at the highest latitude studied $\left(74.3^{\circ} \mathrm{N}\right.$; Zackenberg, Greenland), the daily odds of a caterpillar being attacked by a predator were only $13 \%$ (odds ratio 0.131, confidence limits 0.046 to 0.376 ) of the odds at the equator. Predation rates also decreased with increasing elevation $\left(F_{1,27.1}=6.35, P=\right.$ 0.02; Fig. 1D), independent of latitude (i.e., no latitude by elevation interaction; $F_{1,27.8}=0.70, P=0.41$ ). For every $100 \mathrm{~m}$ moved upward from sea level, the daily odds of predation decreased by $6.6 \%$ (odds ratio 0.934 , confidence limits 0.884 to 0.987 ). At the highest elevation studied (2106 masl, table S1), the daily odds of predation were $24 \%$ of those at sea level (odds ratio 0.238 , confidence limits 0.074 to 0.765$)$.

Notably, higher predation at lower latitudes and elevations was due to more frequent attacks by arthropod predators. The daily odds of a caterpillar suffering an arthropod attack decreased by $3.5 \%$ for every $1^{\circ}$ latitude moved away from the equator (odds ratio 0.966, confidence limits 0.947 to $0.984, F_{1,25.1}=14.11, P<0.001$ ), as did the odds of attack marks that could not be attributed to any specific predator group (odds ratio 0.972 , confidence limits 0.954 to $0.991, F_{1,24.3}=9.57, P=$ 0.005 ; Fig. 1C). By contrast, we found no evidence for a gradient in predation by birds or mammals;

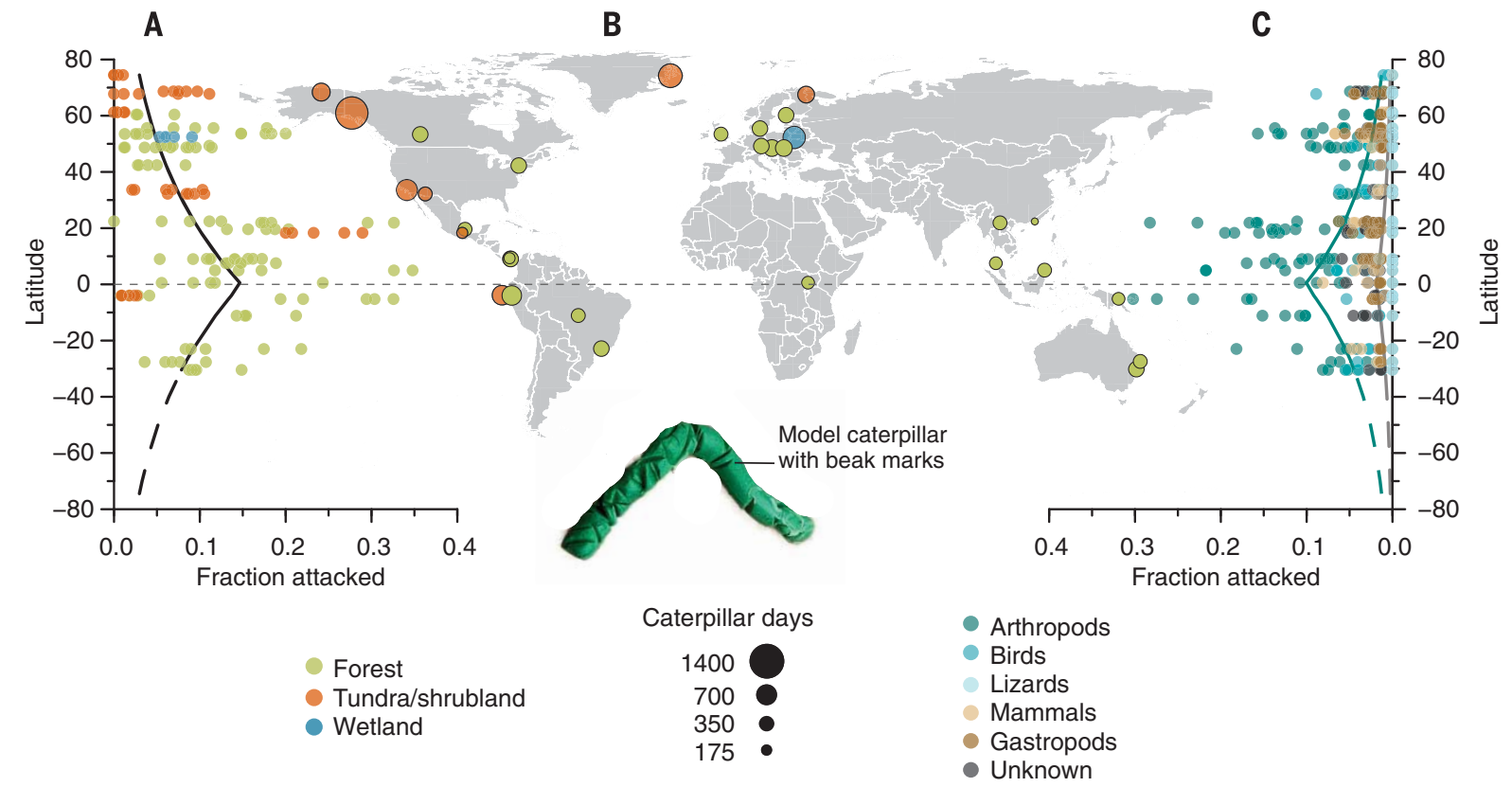

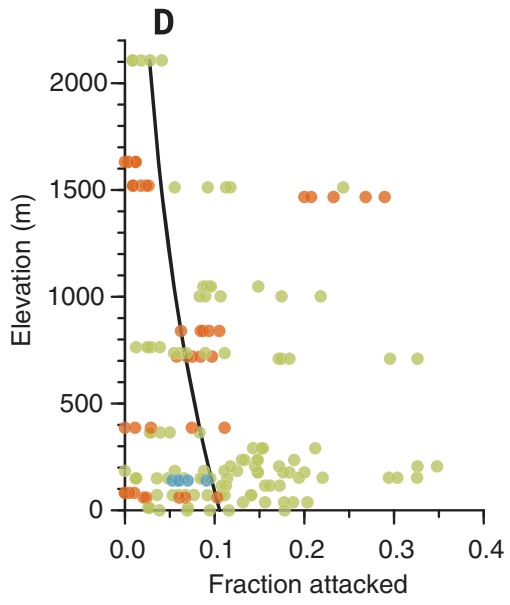

Fig. 1. Map of sampling sites with scatter plots showing fates of model caterpillars at different latitudes and elevations. (A to $\mathbf{F}$ ) In the map of sampling sites across the globe (B) and across elevations (E), individual sites are shown with symbol size graduated by caterpillar days and habitat type identified by the symbol color. For site-specific details, see table S1. (A) and (D) show the overall fraction of caterpillar models attacked per day (i.e., daily predation rates per model caterpillar) in each habitat type, with the patterns resolved by predator type in $(C)$ and $(F)$. Each individual data point reflects the fate of replicate model caterpillars in one of five sampling plots within a
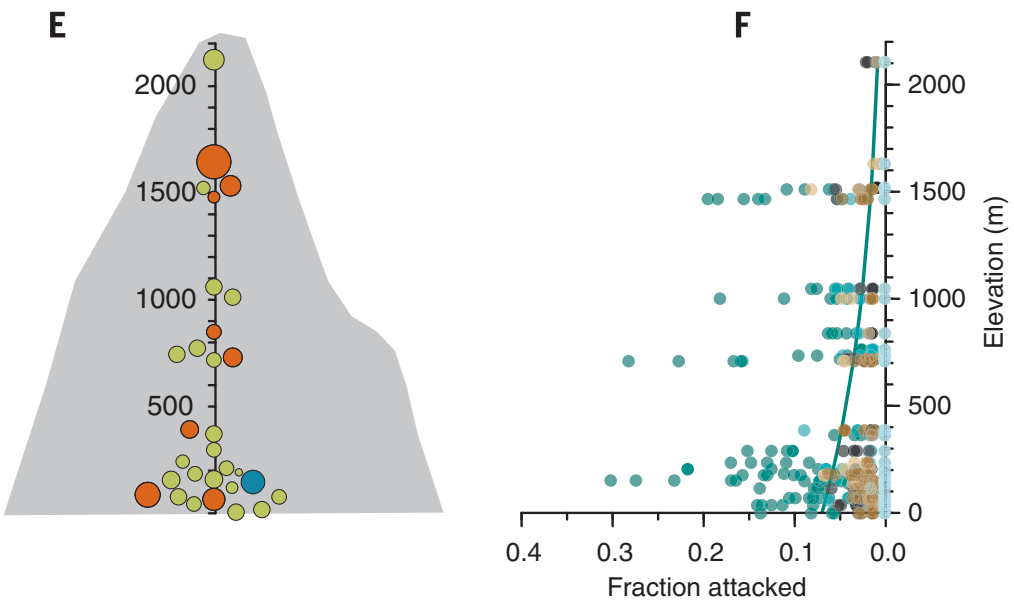
the frequencies of attack marks by these predator groups were unrelated to latitude $\left(F_{1,26.6}=\right.$ $1.20, P=0.28$ and $F_{1,28.6}=2.9, P=0.10$, respectively). These latitudinal patterns in predation rate were mirrored across elevation: The odds of a caterpillar suffering arthropod attack decreased by $9.6 \%$ for every $100 \mathrm{~m}$ moved upward from sea level (odds ratio 0.904, confidence limits 0.839 to $0.975, F_{1,26.1}=7.48, P=0.01$ ), whereas the odds of receiving attack marks not attributable to any specific predator group $\left(F_{1,21.3}=0.18, P=0.68\right)$ or of being attacked by birds $\left(F_{1,29.3}=1.86\right.$, $P=0.18)$ or mammals $\left(F_{1,25.0}=0.63, P=0.44\right)$ were unrelated to elevation (Fig. $1 \mathrm{~F}$ ).

Overall, our study reveals a strong latitudinal and elevational signature on biotic interaction strength (i.e., predation rates) across the globe. In doing so, it provides a clear pattern that can be used to inform future efforts in this field (3-7) and to move beyond the obstacle of contradictory evidence from variable methodologies among studies conducted at different subsets of latitude $(7,13)$. The parallel patterns in predation across elevation [compare (21)] suggest that the ecological factors constraining predation rates are likely to show concordant latitudinal and elevational gradients (34). The clarity of our findings offers a simple lesson: To unmask a global ecological pattern, we may need to apply standardized methods to specific hypotheses determined a priori, rather than combine data derived from different methods a posteriori [compare $(8,13,34)]$. This study thus illustrates the power of simple, low-cost, globally distributed experiments [compare $(17,18,35)]$.

We found that global gradients in predation rate were driven by arthropod predators, with no systematic trend in attack rate by birds or mammals. This latitudinal shift in the relative importance of different predator groups has clear implications for understanding herbivore evolution, interpreting global patterns of herbivory, and understanding global community organization and functioning. In terms of arthropod herbivore evolution, much theory has been developed on latitudinal patterns in plant defense against herbivores, suggesting that if plants at lower latitudes suffer high herbivory, they need to evolve stronger defenses [e.g., $(10,11,15)]$. Our findings motivate an analogous theory for defense deployed by herbivores against their predators. In the tropics, the fraction of model caterpillars attacked per day is notably high (Fig. 1), and attack rates on live prey tend to be even higher (35). Thus, predation in the real world creates a very real selection pressure. This leads to the testable hypothesis that arthropod herbivores in the tropics should be better defended than those at higher latitudes and that these defenses should target arthropod rather than vertebrate predators (Fig. 1C).

Real herbivores accumulate predation risk over their development time, which may be shortened in warmer climates and thus counteract our predictions for the ecological and evolutionary impact of predation gradients. Although a comprehensive assessment goes beyond the current study, we analyzed larval development times from the available literature (table S2), finding a much lower latitudinal effect on development times than on predation rates (table S3). Hence, as a net effect, we expect increased selection pressure on larval herbivores at lower latitudes.

From a plant perspective, the patterns detected in this study suggest increased per capita predation pressure on plant consumers toward the tropics and strong differences in the relative impacts of different predator groups across different regions of the globe (Fig. 1C). This finding suggests markedly different roles for different predator groups in regulating herbivore abundance and traits across geographic gradients, and potential differences in trophic organization between regions. Whether the patterns revealed by the current study translate into patterns in net herbivory is unresolved, particularly considering that our experiments took place in the understory, whereas most primary production takes place in the canopy layer. Although seminal findings suggested latitudinal gradients in herbivory and plant defense $(15,16)$, recent appraisals of the evidence find less support for these trends $(7,8,14)$. Nonetheless, the lack of clear patterns in herbivory may be as much a reflection of variable methods as a true lack of a pattern $(7,13,15,16,36)$. The current study should stimulate further standardized comparisons of species interactions, facilitating a clearer view of these key biological patterns to enlighten our search for their drivers and consequences.

\section{REFERENCES AND NOTES}

1. H. Hillebrand, Am. Nat. 163, 192-211 (2004).

2. J. H. Lawton, Oikos $84,177-192$ (1999).

3. D. W. Schemske, G. G. Mittelbach, H. V. Cornell, J. M. Sobel, K. Roy, Annu. Rev. Ecol. Evol. Syst. 40, 245-269 (2009).

4. D. S. Gruner et al., Ecol. Lett. 11, 740-755 (2008).

5. H. Hillebrand, J. Phycol. 45, 798-806 (2009).

6. J. H. Lambers, J. S. Clark, B. Beckage, Nature 417, 732-735 (2002).

7. A. T. Moles, S. P. Bonser, A. G. B. Poore, I. R. Wallis, W. J. Foley, Funct. Ecol. 25, 380-388 (2011).

8. N. R. Andrew, I. R. Roberts, S. J. Hill, Open J. Ecol. 2, 202-213 (2012)

9. M. Schleuning et al., Curr. Biol. 22, 1925-1931 (2012)
10. T. Dobzhansky, Am. Sci. 38, 209-221 (1950).

11. R. H. MacArthur, Geographical Ecology: Patterns in the Distribution of Species (Princeton Univ. Press, 1972)

12. S. C. Pennings, B. R. Silliman, Ecology 86, 2310-2319 (2005).

13. D. N. Anstett, K. A. Nunes, C. Baskett, P. M. Kotanen, Trends Ecol. Evol. 31, 789-802 (2016)

14. S. Zhang, Y. Zhang, K. Ma, J. Ecol. 104, 1089-1095 (2016)

15. P. D. Coley, T. M. Aide, in Plant-Animal Interactions: Evolutionary Ecology in Tropical and Temperate Regions,

P. W. Price, T. M. Lewinsohn, G. W. Fernandes, W. W. Benson, Eds. (Wiley, 1991), pp. 25-49.

16. P. D. Coley, J. A. Barone, Annu. Rev. Ecol. Syst. 27, 305-335 (1996).

17. L. F. Fraser et al., Front. Ecol. Environ. 11, 147-155 (2013).

18. E. T. Borer et al., Methods Ecol. Evol. 5, 65-73 (2014).

19. P. A. Low, K. Sam, C. McArthur, M. R. C. Posa, D. F. Hochuli, Entomol. Exp. Appl. 152, 120-126 (2014)

20. K. Tvardikova, V. Novotny, J. Trop. Ecol. 28, 331-341 (2012)

21. K. Sam, B. Koane, V. Novotny, Ecography 38, 293-300 (2015).

22. A. J. Lotka, J. Phys. Chem. 14, 271-274 (1910).

23. V. Volterra, Mem. Acad. Lincei Roma 2, 31-113 (1926).

24. P. A. Abrams, Annu. Rev. Ecol. Syst. 31, 79-105 (2000).

25. J. B. Shurin et al., Ecol. Lett. 5, 785-791 (2002).

26. L. Abdala-Roberts, K. A. Mooney, Ecology 95, 2879-2893 (2014)

27. J. S. Bale et al., Glob. Change Biol. 8, 1-16 (2002)

28. C. A. Deutsch et al., Proc. Natl. Acad. Sci. U.S.A. 105 6668-6672 (2008)

29. E. L. Zvereva, M. Kozlov, Glob. Change Biol. 12, 27-41 (2006)

30. E. D. Currano et al., Proc. Natl. Acad. Sci. U.S.A. 105, 1960-1964 (2008)

31. P. Wilf, New Phytol. 178, 486-502 (2008).

32. T. Alerstam, A. Hedenstrom, S. Akesson, Oikos 103, 247-260 (2003).

33. Materials and methods are available as supplementary materials

34. W. K. Petry et al., Science 353, 69-71 (2016).

35. G. L. Lövei, M. Ferrante, Insect Sci. 10.1111/1744-7917.12405 (2016),

36. X. Moreira et al., Ecol. Lett. 17, 537-546 (2014).

\section{ACKNOWLEDGMENTS}

Data are archived at the Dryad digital repository, doi:10.5061/dryad.j432q. We acknowledge funding from the Academy of Finland (grants 138346, 276909, and 285803

to T.R.); European Science Foundation (grant 669609 to V.N.); National Science Foundation (grant OPP 0908502 to A.A and L. Gough, grant DGE-1321846 to C.S.N., and grant 1158817 to E.N.): Instituto de Ecología, A.C. (field work grant to W.D.);

Finnish Cultural Foundation, Oskar Öflunds Stiftelse, and Societas Entomologica Helsingforsiensis (grants to T.H.); Natural Environment Research Council (NERC) (grant NE/ J011169/1 to 0.T.L.); São Paulo Research Foundation (FAPESP) (grant 14/11676-8 to E.N. and grant 13/23457-6 to J. P. Metzger for E.N.); Grant Agency of Czech Republic (grant 14-32024P to K.S., grant 14-04258S to V.N., and grant 14-04258S to A.S.; National Feasibility Program I (L01208 "TEWEP" to A.S.); Estonian Ministry of Education and Research (grant IUT20-33 to T.T.); and Norwegian Research Council's Climate Change and Impacts in Norway (NORKLIMA) program (grant 230607/E10 to V.V.).

\section{SUPPLEMENTARY MATERIALS}

www.sciencemag.org/content/356/6339/742/suppl/DC1

Materials and Methods

Supplementary Text

Fig. S1

Tables $\mathrm{S} 1$ to $\mathrm{S} 3$

References (37-80)

12 September 2016; accepted 6 April 2017

10.1126/science.aai1631 


\section{Science MIAAAS}

Higher predation risk for insect prey at low latitudes and elevations

Tomas Roslin, Bess Hardwick, Vojtech Novotny, William K. Petry, Nigel R. Andrew, Ashley Asmus, Isabel C. Barrio, Yves Basset, Andrea Larissa Boesing, Timothy C. Bonebrake, Erin K. Cameron, Wesley Dáttilo, David A. Donoso, Pavel Drozd, Claudia L. Gray, David S. Hik, Sarah J. Hill, Tapani Hopkins, Shuyin Huang, Bonny Koane, Benita Laird-Hopkins, Liisa Laukkanen, Owen T. Lewis, Sol Milne, Isaiah Mwesige, Akihiro Nakamura, Colleen S. Nell, Elizabeth Nichols, Alena Prokurat, Katerina Sam, Niels M.

Schmidt, Alison Slade, Victor Slade, Alzbeta Suchanková, Tiit

Teder, Saskya van Nouhuys, Vigdis Vandvik, Anita Weissflog, Vital Zhukovich and Eleanor M. Slade (May 18, 2017)

Science 356 (6339), 742-744. [doi: 10.1126/science.aaj1631]

Editor's Summary

\section{Risky in the tropics}

It is well known that diversity increases toward the tropics. Whether this increase translates into differences in interaction rates among species, however, remains unclear. To simplify the problem, Roslin et al. tested for predation rates by using a single approach involving model caterpillars across six continents. Predator attack rates were higher toward the equator, but only for arthropod predators.

Science, this issue p. 742

This copy is for your personal, non-commercial use only.

Article Tools Visit the online version of this article to access the personalization and article tools:

http://science.sciencemag.org/content/356/6339/742

Permissions Obtain information about reproducing this article: http://www.sciencemag.org/about/permissions.dtl

Science (print ISSN 0036-8075; online ISSN 1095-9203) is published weekly, except the last week in December, by the American Association for the Advancement of Science, 1200 New York Avenue NW, Washington, DC 20005. Copyright 2016 by the American Association for the Advancement of Science; all rights reserved. The title Science is a registered trademark of AAAS. 